\title{
SEMI-QUADRATIC VARIATIONAL PROBLEMS FOR MULTIPHASE EQUILIBRIA.
}

\author{
BY \\ ELIOT FRIED (Dept. of Theoretical and Applied Mechanics, University of Illinois, Urbana) \\ AND \\ MORTON E. GURTIN (Dept. of Mathematics, Carnegie Mellon University, Pittsburgh, Pennsylvania)
}

1. Introduction. Mechanical approaches to the study of multiphase equilibria in solids are most often based on the infinitesimal and finite theories of elasticity. ${ }^{1}$ However:

(i) The infinitesimal theory does not allow for finite transition strains between phases, and cannot account for the large rotations that generally accompany twinning.

(ii) The finite theory accounts for both large strains and large rotations, but requires a model strain-energy (density) to compute actual material behavior; because of the difficulties inherent in the characterization of real materials at large strains and rotations, the development of such models is in its infancy. ${ }^{2}$

In this note we take a completely different approach. While we see the need to allow for finite strains, we confront the difficulty of obtaining accurate models by limiting our discussion to behavior in which the strain at any point lies close to a value corresponding a local minimum of the strain energy, although different points might correspond to different minima. Nothing is assumed regarding the size of the rotation or the size of the transition strains between local minima. ${ }^{3}$

More precisely, we consider a strain energy $\widetilde{W}(\boldsymbol{E})$ with local minima at $\boldsymbol{E}=\boldsymbol{E}_{q}$ $(q=1,2, \ldots, Q)$, and approximate $\widetilde{W}(\boldsymbol{E})$ by a sum of quadratic wells centered at the $\boldsymbol{E}_{q}$; here $\boldsymbol{E}$ is the finite strain measure ${ }^{4}$

$$
\boldsymbol{E}=\frac{1}{2}\left(\nabla \boldsymbol{u}+\nabla \boldsymbol{u}^{\mathrm{T}}+\nabla \boldsymbol{u}^{\mathrm{T}} \nabla \boldsymbol{u}\right)
$$

\footnotetext{
Received July 1, 1993.

1991 Mathematics Subject Classification. Primary 49H05, 73G05; Secondary 73C05, 73C60.

${ }^{\prime}$ For work based on infinitesimal elasticity, cf. [1-11]. For work based on finite elasticity, cf. [12-30]. For a comparison of the infinitesimal and finite approaches, cf. [31].

${ }^{2} \mathrm{Cf}$. Ball and James [32].

${ }^{3}$ In this sense our theory is the same as that developed by Grinfeld [33], whose paper we discovered after completing this work. However, the thrust of our work differs from that of [33]: Grinfeld begins with balance laws and discusses problems involving inclusions, while we focus on the variational characterization of equilibria, with and without interfacial energy, allowing for a sharp interface or a transition layer.

${ }^{4}$ For the most part we use the notation of [34]; in particular, 1 is the identity tensor, while $\boldsymbol{F}^{\mathrm{T}}$ designates the transpose of a tensor $\boldsymbol{F}$.
} 
with $\boldsymbol{u}$ the displacement. Using this ansatz, we arrive at an "approximate" strain energy

$$
W(\boldsymbol{E}, \boldsymbol{\chi})=\sum_{q=1}^{Q} \chi_{q} W_{q}(\boldsymbol{E}),
$$

with

$$
W_{q}(\boldsymbol{E})=w_{q}+\frac{1}{2}\left(\boldsymbol{E}-\boldsymbol{E}_{q}\right) \cdot \mathbb{A}_{q}\left[\boldsymbol{E}-\boldsymbol{E}_{q}\right], \quad w_{q}=W\left(\boldsymbol{E}_{q}\right) .
$$

Here:

(i) $\mathbb{A}_{q}[\cdot]$-a linear transformation from the space of symmetric tensors into itselfis the second derivative of $\widetilde{W}(\boldsymbol{E})$ at $\boldsymbol{E}=\boldsymbol{E}_{q}$ and can be determined knowing only the elasticity tensor appropriate to infinitesimal deformations from a reference configuration at strain $\boldsymbol{E}_{q}$.

(ii) $\chi=\left(\chi_{1}, \chi_{2}, \ldots, \chi_{Q}\right)$ is a pure phase; that is, one of its entries has value 1, while all of its other entries vanish. Let $\chi=\chi(x)$ and $\boldsymbol{E}=\boldsymbol{E}(\boldsymbol{x})$. Then, at points $\boldsymbol{x}$ with $\chi_{q}(\boldsymbol{x})=1$ the strain energy $W(\boldsymbol{E}, \boldsymbol{\chi})$ reduces to the energy $W_{q}(\boldsymbol{E})$ appropriate to behavior near $\boldsymbol{E}_{q}$; in this sense, for $\Omega$ the region of space occupied by the body,

$$
\Omega_{q}=\left\{\boldsymbol{x} \in \Omega: \chi_{q}(\boldsymbol{x})=1\right\}
$$

represents the region occupied by phase $q$, with $\chi_{q}$ its characteristic function.

We limit our discussion to the formal deduction of appropriate variational problems for the characterization of equilibria; we do not attempt the corresponding analysis. The simplest such problem is the semi-quadratic variational problem

$$
\operatorname{minimize}_{\boldsymbol{u} \in \mathscr{U}_{s}, \boldsymbol{\chi} \in \mathscr{\mathscr { \ell }}} \int_{\Omega} W(\boldsymbol{E}, \boldsymbol{\chi}) d v+\boldsymbol{\Phi}(\boldsymbol{u}),
$$

where $\mathscr{U}_{s}$ is the set of coherent (continuous) displacement fields consistent with the displacement boundary-conditions and a requirement of rank-one convexity of the individual wells $W_{q}(\boldsymbol{E})$ (cf. (4.11)), $\Phi(\boldsymbol{u})$ is the joint potential of the surface tractions and body force, and $\mathscr{X}$ is the set of functions $\chi$ on $\Omega$ with $\chi(x)$ a pure phase at each $\boldsymbol{x} \in \Omega$.

We also give a generalization of (1.5) that includes interfacial energy, as well as a regularization of (1.5) in which $\chi$ is replaced by an order parameter (phase-field), with surface energy modeled by a dependence of energy on $\nabla \chi$.

In an Appendix we give formal derivations of the Euler-Lagrange equations (bulk equations and interface conditions) associated with the variational principles under consideration.

\section{General formulation.}

a. Kinematics. Throughout this note $\Omega$ is a body identified with the region of space it occupies in a fixed reference configuration. A displacement of $\Omega$ is then a vector field $\boldsymbol{u}$ on $\Omega$ with deformation gradient

$$
\boldsymbol{F}=\mathbf{1}+\nabla \boldsymbol{u}
$$

consistent with $\operatorname{det} \boldsymbol{F}>0$. The polar decomposition

$$
\boldsymbol{F}=\boldsymbol{R} \boldsymbol{U}
$$


allows a decomposition of $\boldsymbol{F}$ into a pure strain

$$
\boldsymbol{U}=\left(\boldsymbol{F}^{\mathrm{T}} \boldsymbol{F}\right)^{1 / 2},
$$

called the stretch, followed by a rotation $\boldsymbol{R}$. Here we find it convenient to use, in place of $\boldsymbol{U}$, the Green strain

$$
\boldsymbol{E}=\frac{1}{2}\left(\boldsymbol{U}^{2}-\mathbf{1}\right)=\frac{1}{2}\left(\boldsymbol{F}^{\mathrm{T}} \boldsymbol{F}-\mathbf{1}\right)=\frac{1}{2}\left(\nabla \boldsymbol{u}+\nabla \boldsymbol{u}^{\mathrm{T}}+\nabla \boldsymbol{u}^{\mathrm{T}} \nabla \boldsymbol{u}\right) .
$$

When $\nabla \boldsymbol{u}$ is small, $\boldsymbol{E}$ is approximated by the infinitesimal strain tensor

$$
\frac{1}{2}\left(\nabla \boldsymbol{u}+\nabla \boldsymbol{u}^{\mathrm{T}}\right)
$$

upon which the linear theory of elasticity is based; here we will not assume that $\nabla u$ is small, only that $\boldsymbol{E}$ is close to a discrete set consisting of local minima of the strain energy.

b. Strain energy. We consider an elastic material with strain energy $\widetilde{W}(\boldsymbol{E})$ a function of $\boldsymbol{E}$. Its derivative $D \widetilde{W}(\boldsymbol{E})$ with respect to $\boldsymbol{E}$ is then a symmetric tensor, while its second derivative $D^{2} \widetilde{W}(\boldsymbol{E})$ is a linear transformation of symmetric tensors $\boldsymbol{V}$ into symmetric tensors $D^{2} \widetilde{W}(\boldsymbol{E})[\boldsymbol{V}]$. By (2.4), the (Piola-Kirchhoff) stress $\boldsymbol{S}$, which is the derivative of $\widetilde{W}(\boldsymbol{E})$ with respect to $\boldsymbol{F}$, is given by

$$
\tilde{\boldsymbol{S}}(\boldsymbol{F})=\boldsymbol{F} D \widetilde{W}(\boldsymbol{E}) .
$$

We assume that $\widetilde{W}(\boldsymbol{E})$ has local minima $\boldsymbol{E}=\boldsymbol{E}_{q}(q=1,2, \ldots, Q)$; then, writing

$$
\mathbb{A}_{q}:=D^{2} \widetilde{W}\left(\boldsymbol{E}_{q}\right)
$$

we see that

$$
D \widetilde{W}\left(\boldsymbol{E}_{q}\right)=0, \quad \mathbb{A}_{q} \text { is positive semi-definite. }
$$

We will refer to $q$ as the phase, even though some $q$-values might correspond to variants of a given phase.

c. Determining $\mathbb{A}_{q}$ from the elasticity tensor $\mathbb{C}_{q}$ for infinitesimal deformations from phase $q$. We write $\boldsymbol{U}_{q}$ for the stretch tensor corresponding to $\boldsymbol{E}_{q}$ :

$$
\boldsymbol{E}_{q}=\frac{1}{2}\left(\boldsymbol{U}_{q}^{2}-\mathbf{1}\right)
$$

Suppose that we deform the body from the reference configuration by first stretching it with deformation gradient $\boldsymbol{U}_{q}$ and then stretching it again, from this deformed configuration, with stretch $\boldsymbol{G}$; the total deformation gradient $\boldsymbol{F}$ and stretch $\boldsymbol{U}$ are then given by

$$
\boldsymbol{F}=\boldsymbol{G} \boldsymbol{U}_{q}, \quad \boldsymbol{U}^{2}=\boldsymbol{U}_{q} \boldsymbol{G}^{2} \boldsymbol{U}_{q},
$$

and

$$
\boldsymbol{G}^{2}=\mathbf{1}+\nabla \boldsymbol{g}+\nabla \boldsymbol{g}^{\mathrm{T}}+\nabla \boldsymbol{g}^{\mathrm{T}} \nabla \boldsymbol{g}
$$

with $\boldsymbol{g}$ the associated displacement. by

The strain energy-as a function $\mathscr{W}_{q}(\boldsymbol{G})$ of the stretch $\boldsymbol{G}$ from phase $q$-is given

$$
\mathscr{W}_{q}(\boldsymbol{G})=\widetilde{W}(\boldsymbol{E}), \quad \boldsymbol{E}=\frac{1}{2}\left(\boldsymbol{U}_{q} \boldsymbol{G}^{2} \boldsymbol{U}_{q}-\mathbf{1}\right)
$$


In view of $(2.8)$,

$$
D \mathscr{W}_{q}(\mathbf{1})=\mathbf{0}
$$

and, since

$$
\boldsymbol{G}=\mathbf{1}+\frac{1}{2}\left(\nabla \boldsymbol{g}+\nabla \boldsymbol{g}^{\mathrm{T}}\right)+\boldsymbol{O}\left(|\nabla \boldsymbol{g}|^{2}\right),
$$

with $\frac{1}{2}\left(\nabla \boldsymbol{g}+\nabla \boldsymbol{g}^{\mathrm{T}}\right)$ the infinitesimal strain from phase $q$, the linear transformation

$$
\mathbb{C}_{q}:=D^{2} \mathscr{W}_{q}(\mathbf{1})
$$

of symmetric tensors into symmetric tensors represents the elasticity tensor for infinitesimal deformations from $q$. If we compute the second derivative of $(2.12)$ with respect to $\boldsymbol{G}$ at $\boldsymbol{G}=\mathbf{1}$, by taking $\boldsymbol{G}=\mathbf{1}+\alpha \boldsymbol{B}+\beta \boldsymbol{H}$, with $\boldsymbol{B}$ and $\boldsymbol{H}$ symmetric tensors, and then computing $\partial^{2} \mathscr{W}_{q}(\boldsymbol{G}) / \partial \alpha \partial \beta$ at $\alpha=\beta=0$, we find that

$$
\boldsymbol{B} \cdot \mathbb{C}_{q}[\boldsymbol{H}]=\left(\boldsymbol{U}_{q} \boldsymbol{B} \boldsymbol{U}_{q}\right) \cdot \mathbb{A}_{q}\left[\boldsymbol{U}_{q} \boldsymbol{H} \boldsymbol{U}_{q}\right]
$$

and, since $\boldsymbol{B}$ and $\boldsymbol{H}$ are arbitrary,

$$
\mathbb{C}_{q}[\boldsymbol{H}]=\boldsymbol{U}_{q} \mathbb{A}_{q}\left[\boldsymbol{U}_{q} \boldsymbol{H} \boldsymbol{U}_{q}\right] \boldsymbol{U}_{q}
$$

for all $\boldsymbol{H}$, so that

$$
\mathbb{A}_{q}[\boldsymbol{M}]=\boldsymbol{V}_{q} \mathbb{C}_{q}\left[\boldsymbol{V}_{q} \boldsymbol{M} \boldsymbol{V}_{q}\right] \boldsymbol{V}_{q}, \quad \boldsymbol{V}_{q}=\boldsymbol{U}_{q}^{-1}
$$

for all symmetric tensors $\boldsymbol{M}$. If we denote the Cartesian components ${ }^{5}$ of $\mathbb{C}_{q}, \mathbb{A}_{q}$, and $V_{q}$ by $C_{i j k l}, A_{i j k l}$, and $V_{k l}$, then

$$
A_{i j k l} M_{k l}=V_{a i} V_{b j} C_{a b k l} V_{k r} V_{l s} M_{r s}
$$

for all symmetric $\boldsymbol{M}$.

Thus $\mathbb{A}_{q}$ can be determined knowing only $U_{q}$ and the elasticity tensor $\mathbb{C}_{q}$ for infinitesimal deformations from phase $q$.

3. Variational characterization of equilibrium. Our discussion is completely formal: we will not specify regularity hypotheses other than to note that the displacement is required to be coherent (continuous and piecewise smooth).

We consider the equilibrium of $\Omega$ under loading conditions for which the displacement satisfies $\boldsymbol{u}=\hat{\boldsymbol{u}}$ on a portion $S$ of $\partial \Omega$, and for which the surface tractions on the remainder of $\partial \Omega$ and the body force $\boldsymbol{b}$ are derived from a potential $\Phi(\boldsymbol{u})$. Writing

$$
\mathscr{U}=\{\text { coherent } \boldsymbol{u}: \boldsymbol{u}=\hat{\boldsymbol{u}} \text { on } S, \operatorname{det}(\mathbf{1}+\nabla \boldsymbol{u})>0 \text { in } \Omega\},
$$

we consider the exact variational problem

$$
\underset{\boldsymbol{u} \in \mathscr{U}}{\operatorname{minimize}} \int_{\Omega} \widetilde{W}(\boldsymbol{E}) d v+\boldsymbol{\Phi}(\boldsymbol{u})
$$

The requirement of coherency yields the standard compatibility condition

$$
[\boldsymbol{F}]=\boldsymbol{a} \otimes \boldsymbol{n}
$$

\footnotetext{
${ }^{5}$ That is, e.g., $\boldsymbol{T}=\mathbb{C}_{q}[\boldsymbol{M}]$ if and only if $T_{i j}=C_{i j k l} M_{k l}$ (using the standard notation of Cartesian tensor analysis with summation convention).
} 
across a surface $\mathscr{S}$ of discontinuity, giving the jump in $\boldsymbol{F}$ in terms of the normal $\boldsymbol{n}$ to $\mathscr{S}$ and $\boldsymbol{a}=[\boldsymbol{F}] \boldsymbol{n}$. (Our convention is that $[\boldsymbol{F}]$ is the limit from the region into which $n$ points minus that from the other region.) The Euler-Lagrange equations corresponding to the exact variational problem consist of the stress equation of equilibrium

$$
\operatorname{div} \tilde{\boldsymbol{S}}(\boldsymbol{F})+\boldsymbol{b}=\mathbf{0}
$$

on regions over which the displacement is smooth in conjunction with the jump conditions

$$
[\widetilde{\boldsymbol{S}}(\boldsymbol{F}) \boldsymbol{n}]=\mathbf{0}, \quad[\widetilde{W}(\boldsymbol{E})-\widetilde{\boldsymbol{S}}(\boldsymbol{F}) \cdot \boldsymbol{F}]=0
$$

across surfaces of discontinuity (enforcing continuity of the surface traction $\widetilde{\boldsymbol{S}}(\boldsymbol{F}) \boldsymbol{n}$ and Eshelby traction $\left.\left(\widetilde{W}(\boldsymbol{E}) \mathbf{1}-\boldsymbol{F}^{\mathrm{T}} \widetilde{\boldsymbol{S}}(\boldsymbol{F})\right) \boldsymbol{n}\right)$.

4. Behavior near potential wells. The semi-quadratic variational problem. We are interested in the behavior of $\widetilde{W}(\boldsymbol{E})$ near its local minima at $\boldsymbol{E}=\boldsymbol{E}_{q} \quad(q=$ $1,2, \ldots, Q)$. Choosing a particular phase $q$ and expanding $\widetilde{W}(\boldsymbol{E})$ about $\boldsymbol{E}=\boldsymbol{E}_{q}$, we find, using (2.7) and (2.8), that

$$
\widetilde{W}(E)=\widetilde{W}\left(\boldsymbol{E}_{q}\right)+\frac{1}{2}\left(\boldsymbol{E}-\boldsymbol{E}_{q}\right) \cdot \mathbb{A}_{q}\left[\boldsymbol{E}-\boldsymbol{E}_{q}\right]+O\left(\left|\boldsymbol{E}-\boldsymbol{E}_{q}\right|^{3}\right) .
$$

Therefore, to within terms of $O\left(\left|\boldsymbol{E}-\boldsymbol{E}_{q}\right|^{3}\right)$, the strain energy is approximated by the quadratic form

$$
W_{q}(\boldsymbol{E})=w_{q}+\frac{1}{2}\left(\boldsymbol{E}-\boldsymbol{E}_{q}\right) \cdot \mathbb{A}_{q}\left[\boldsymbol{E}-\boldsymbol{E}_{q}\right], \quad w_{q}=\widetilde{W}\left(\boldsymbol{E}_{q}\right),
$$

which has associated with it the stress

$$
\boldsymbol{S}_{q}(\boldsymbol{F})=\boldsymbol{F} D W_{q}(\boldsymbol{E})=\boldsymbol{F} \mathbb{A}_{q}\left[\boldsymbol{E}-\boldsymbol{E}_{q}\right] .
$$

We assume that $\mathbb{A}_{q}$, as a linear transformation of symmetric tensors into symmetric tensors, is positive definite. Even so, the nonlinear dependence of $\boldsymbol{E}$ on $\boldsymbol{F}$ generally results in a loss of rank-one convexity for $W_{q}(\boldsymbol{E})$ as a function of $\boldsymbol{F}$. In fact, a straightforward calculation shows that $W_{q}(E)$ is strictly rank-one convex as a function of $\boldsymbol{F}$ if and only if the inequality

$$
(\boldsymbol{a} \otimes \boldsymbol{a}) \cdot \mathbb{A}_{q}\left[\boldsymbol{E}-\boldsymbol{E}_{q}\right]+(\boldsymbol{a} \otimes \boldsymbol{U} \boldsymbol{b}) \cdot \mathbb{A}_{q}[\boldsymbol{a} \otimes \boldsymbol{U} \boldsymbol{b}]>0
$$

holds for all unit vectors $\boldsymbol{a}$ and $\boldsymbol{b}$. We note that the set of symmetric tensors satisfying (4.4) is open, and since $\mathbb{A}_{q}$ is positive definite, this set contains $\boldsymbol{E}_{q}$. When $\mathbb{A}_{q}$ is isotropic with Lamé modulus $\lambda_{q}$ and shear modulus $\mu_{q}$, the left-hand side of (4.4) has a particularly simple form:

$$
\lambda_{q}\left\{\operatorname{tr}\left(\boldsymbol{E}-\boldsymbol{E}_{q}\right)+(\boldsymbol{a} \cdot \boldsymbol{U} \boldsymbol{b})^{2}\right\}+2 \mu_{q}\left\{\boldsymbol{a} \cdot\left(\boldsymbol{E}-\boldsymbol{E}_{q}\right) \boldsymbol{a}+\frac{1}{2}|\boldsymbol{U} \boldsymbol{b}|^{2}+\frac{1}{2}(\boldsymbol{a} \cdot \boldsymbol{U b})^{2}\right\},
$$

so that $(4.4)$ is satisfied provided

$$
\lambda_{q} \operatorname{tr}\left(\boldsymbol{E}-\boldsymbol{E}_{q}\right)+\frac{1}{2} \mu_{q}\left\{\boldsymbol{a} \cdot\left(\boldsymbol{E}-\boldsymbol{E}_{q}\right) \boldsymbol{a}+\frac{1}{2}|\boldsymbol{U} \boldsymbol{b}|^{2}\right\}>0 .
$$

If we denote the least eigenvalue of $\boldsymbol{E}-\boldsymbol{E}_{q}$ by $k_{q}$, and the smallest principal stretch associated with $U$ by $h$, then (4.6) holds whenever

$$
\lambda_{q} \operatorname{tr}\left(\boldsymbol{E}-\boldsymbol{E}_{q}\right)+2 \mu_{q} k_{q}+\mu_{q} h^{2}>0 .
$$


By choosing $a$ to coincide with the smallest principal stretch and $b$ orthogonal to $\boldsymbol{U a}$, we see that (4.7) is also necessary for (4.6).

We now consider a coherent displacement field and assume that, at any given $\boldsymbol{x} \in$ $\Omega, \boldsymbol{E}(\boldsymbol{x})$ is close to exactly one of the natural strains $\boldsymbol{E}_{q}$ (although the appropriate natural strain may vary from point to point). Further, for each $\boldsymbol{x}$, we let $\chi(\boldsymbol{x})$ be the pure phase (defined in Sec. 1) that has $\chi_{q}(\boldsymbol{x})=1$ when $\boldsymbol{E}_{q}$ is the appropriate natural strain at $\boldsymbol{x}$. Then, to within terms of order

$$
O\left(\sum_{q=1}^{Q} \chi_{q}\left|\boldsymbol{E}-\boldsymbol{E}_{q}\right|^{3}\right),
$$

we can approximate the strain energy by

$$
W(\boldsymbol{E}, \boldsymbol{\chi})=\sum_{q=1}^{Q} \chi_{q} W_{q}(\boldsymbol{E}),
$$

with

$$
\boldsymbol{S}(\boldsymbol{F}, \boldsymbol{\chi})=\sum_{q=1}^{Q} \chi_{q} \boldsymbol{S}_{q}(\boldsymbol{F})
$$

the corresponding stress. Further, to ensure rank-one convexity we restrict attention to displacement fields belonging to the set

$$
\mathscr{U}_{s}=\{\boldsymbol{u} \in \mathscr{U} \text { : at each } \boldsymbol{x} \in \Omega, \boldsymbol{E}(\boldsymbol{x}) \text { satisfies (4.4) for some } q\} .
$$

Letting $\mathscr{X}$ denote the set of sufficiently regular functions $\chi$ with $\chi(x)$ a pure phase at each $\boldsymbol{x}$, we are led to the semi-quadratic variational problem:

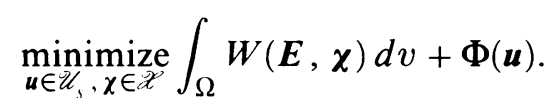

The Euler-Lagrange equations for (4.12) are (3.4)-(3.5) with $\widetilde{W}(\boldsymbol{E})$ and $\widetilde{\boldsymbol{S}}(\boldsymbol{F})$ replaced by $\boldsymbol{W}(\boldsymbol{E}, \boldsymbol{\chi})$ and $\boldsymbol{S}(\boldsymbol{F}, \boldsymbol{\chi})$.

REMARKs. 1. If, for a solution $(\boldsymbol{u}, \boldsymbol{\chi})$ of this problem, the strain $\boldsymbol{E}$ takes on values corresponding only to the minima $\boldsymbol{E}_{q}$ of the strain energy, then $\boldsymbol{u}$ will also be a solution of the exact variational problem (3.2). The extent to which the semiquadratic problem approximates the exact problem is an interesting open question.

2. As shown in Sec. $2 c, \mathbb{A}_{q}$ can be determined knowing only $\boldsymbol{U}_{q}$ and the elasticity tensor $\mathbb{C}_{q}$ for infinitesimal deformations from phase $q$. Since $\mathbb{C}_{q}$ is known for a large class of materials, the strain energy $W(\boldsymbol{E}, \boldsymbol{\chi})$ is, in principle, not difficult to determine.

3. In the variational problem (4.12) the underlying restriction,

$$
\left|\boldsymbol{E}(\boldsymbol{x})-\boldsymbol{E}_{q}\right| \text { is small when } \chi_{q}(\boldsymbol{x})=1,
$$

is to be verified a posteriori. This variational problem is, however, meaningful irrespective of (4.13) and might play a role in the study of phase transitions similar to that played by the linear theory of elasticity in more classical settings. (With proper interpretation, linear elasticity is successfully used to study phenomena such as fracture, even though it yields infinite strain at the tip of a sharp crack.) 
Surface energy for interfaces between phases can be defined formally through the measure

$$
\Gamma(\nabla \chi)=\frac{1}{4} \sum_{q, p=1}^{Q} \sigma_{q p}\left\{\left|\nabla \chi_{q}\right|+\left|\nabla \chi_{p}\right|-\left|\nabla\left(\chi_{q}+\chi_{p}\right)\right|\right\},
$$

where $\sigma_{q p}=\sigma_{p q}$ are constants with $\sigma_{q p}>0$ if $q \neq p$ and $\sigma_{q q}=0 ; \sigma_{q p}$ represents the surface energy (per unit area) of the interface $\mathscr{S}_{q p}$ between phases $q$ and $p$. $\Gamma(\nabla \chi)$ vanishes away from such interfaces, while $\Gamma(\nabla \chi)=\sigma_{q p}\left|\nabla \chi_{q}\right|$ on $\mathscr{S}_{q p}$ (since $\chi_{q}+\chi_{p}$ is continuous across $\mathscr{S}_{q p}$ and $\left|\nabla \chi_{q}\right|=\left|\nabla \chi_{p}\right|$ on $\left.\mathscr{S}_{q p}\right)$.

The semi-quadratic variational problem with interfacial energy takes the form:

$$
\operatorname{minimize}_{\boldsymbol{u} \in \mathscr{U}_{s}, \boldsymbol{\chi} \in \mathscr{\mathscr { X }}} \int_{\Omega}\{\boldsymbol{W}(\boldsymbol{E}, \boldsymbol{\chi})+\Gamma(\nabla \boldsymbol{\chi})\} d v+\boldsymbol{\Phi}(\boldsymbol{u}) .
$$

The formal Euler-Lagrange equations corresponding to this variational problem consist of the stress equation of equilibrium (3.4) with $\tilde{\boldsymbol{S}}(\boldsymbol{F})$ replaced by $\boldsymbol{S}(\boldsymbol{F}, \chi)$ in each of the phase regions $\Omega_{q}$ in conjunction with jump conditions

$$
[\boldsymbol{S}(\boldsymbol{F}, \boldsymbol{\chi}) \boldsymbol{n}]=0, \quad[\boldsymbol{W}(\boldsymbol{E}, \boldsymbol{\chi})-\boldsymbol{S}(\boldsymbol{F}, \boldsymbol{\chi}) \cdot \boldsymbol{F}]=-2 \sigma_{q p} K
$$

across each interface $\mathscr{S}_{q p}$, with $n$ the unit normal to $\partial \Omega_{q}$ and $K$ twice the mean curvature of $\mathscr{S}_{q p}$ (taken as positive whenever the osculating sphere lies in $\Omega_{q}$ ). An additional interface condition is supplied by the coherency relation (3.3).

5. Regularized theory. We now consider a regularized theory in which the $\chi_{q}$ act as phase fractions and interfaces are identified with thin transition zones throughout which $\chi$ exhibits large gradients.

More precisely, we no longer require that $\chi$ be a pure phase, but instead allow $\chi$ to take on any value in $\mathbb{R}^{Q}$ consistent with the constraints

$$
\sum_{q=1}^{Q} \chi_{q}=1, \quad \chi_{q} \in[0,1] \text { for each } q .
$$

We retain the definitions (4.9) and (4.10) for the strain energy $W(\boldsymbol{E}, \boldsymbol{\chi})$ and stress $\boldsymbol{S}(\boldsymbol{F}, \boldsymbol{\chi})$, so that, by $(5.1), \chi_{q}$ may be interpreted as the volume fraction of phase $q$.

Recognizing that in the current context each well of the potential is generally operative at each point in $\Omega$, we restrict attention to displacement fields that belong to

$$
\mathscr{U}_{r}=\{\boldsymbol{u} \in \mathscr{U} \text { : at each } \boldsymbol{x} \in \Omega, \boldsymbol{E}(\boldsymbol{x}) \text { satisfies (4.4) for all } q\} .
$$

We assume that this set is not empty. In the case of $Q$ isotropic phases the requirement that $\mathscr{U}_{r}$ be nonempty is

$$
\lambda_{q} \operatorname{tr}\left(\boldsymbol{E}_{p}-\boldsymbol{E}_{q}\right)+2 \mu_{q} k_{p q}+\mu_{q} h_{p}^{2}>0
$$

for all $p$ and $q$, where $k_{p q}$ denotes the minimum eigenvalue of $\boldsymbol{E}_{p}-\boldsymbol{E}_{q}$, and $h_{p}$ is the minimum eigenvalue of $\boldsymbol{U}_{p}$. 
At equilibrium we want $\chi$ to be near the set of pure phases; with this in mind, we introduce on

$$
\mathscr{P}=\left\{\chi \in \mathbb{R}^{Q}: \chi \text { satisfies the constraints }(5.1)\right\}
$$

an exchange energy $f(\chi)$ with

$$
f(\chi)>0 \text { for } \chi \neq \chi_{q}, q=1,2, \ldots, q ; \quad f\left(\chi_{q}\right)=0 .
$$

Because of $(5.1)$,

$$
\sum_{q=1}^{Q} \nabla \chi_{q}=0
$$

Taking this into account, we model interfacial energy using a gradient energy,

$$
g(\nabla \boldsymbol{\chi})=\frac{1}{2} \sum_{q, p=1}^{Q} \lambda_{q p} \nabla \chi_{q} \cdot \nabla \chi_{p},
$$

where $\lambda_{q p}=\lambda_{p q}$ are constants, where

$$
\sum_{s=1}^{Q} \lambda_{q s}=0
$$

and where the matrix with entries $\lambda_{q p}$ is positive definite on the subspace of vectors in $\mathbb{R}^{Q}$ whose entries sum to zero.

Let $\overline{\mathscr{X}}$ denote the set of all sufficiently regular functions $\chi: \Omega \rightarrow \mathscr{P}$. The regularized variational problem then has the form

$$
\operatorname{minimize}_{\boldsymbol{u} \in \mathscr{U}_{r}, \boldsymbol{x} \in \overline{\mathscr{X}}} \int_{\Omega}\{W(\boldsymbol{E}, \boldsymbol{\chi})+f(\boldsymbol{\chi})+g(\nabla \boldsymbol{\chi})\} d v+\boldsymbol{\Phi}(\boldsymbol{u}) .
$$

The Euler-Lagrange equations corresponding to (5.9) consist of a stress equation of equilibrium

$$
\operatorname{div} \boldsymbol{S}(\boldsymbol{F}, \boldsymbol{\chi})+\mathbf{b}=0
$$

in conjunction with an equation

$$
\sum_{p=1}^{Q} \lambda_{q p} \Delta \chi_{p}+\pi_{q}=0
$$

for each phase $q=1,2, \ldots, Q$, where

$$
\pi_{q}=-\partial_{\chi_{q}} f(\boldsymbol{\chi})-W_{q}(\boldsymbol{E})+(1 / Q) \sum_{p=1}^{Q} W_{p}(\boldsymbol{E}),
$$

and where $\partial_{\chi_{u}} f$ represents the " $q$-component" of the gradient of $f$ on the hyperplane defined by the first of (5.1), so that

$$
\sum_{q=1}^{Q} \partial_{\chi_{q}} f(x)=0
$$


To qualify as a regularization of (4.12) the total energy should be dominated by contributions from the strain energy in regions of pure phase and by the exchange and gradient energies between such regions. To achieve this the different terms of the energy must scale appropriately. Specifically, suppose $\Omega$ contains a single interface of dimensionless thickness $\varepsilon$ that separates two phases. Let $l$ be a characteristic length associated with $\Omega$, and let $\mu, \nu$, and $\lambda$ denote scale factors for $W, f$, and $g$, respectively. Provided these obey $\mu / \nu=O(\varepsilon)$ and $\lambda /\left(\nu l^{2}\right)=O\left(\varepsilon^{2}\right)$, an argument used in [35] can be adapted to show that the Euler-Lagrange equations of (5.9) tend formally, as $\varepsilon \rightarrow 0$, to those of (4.12). This argument yields, furthermore, a relationship between the coefficient $\sigma_{p q}$ used in the sharp interface theory, and the exchange and gradient energy terms used in the regularized theory.

The problem (5.9) has a simple generalization: in place of $W(\boldsymbol{E}, \boldsymbol{\chi})+f(\boldsymbol{\chi})+$ $g(\nabla \chi)$ we consider an energy $\bar{W}(\boldsymbol{E}, \chi, \nabla \chi)$, that need not be semi-quadratic, in conjunction with the problem:

$$
\operatorname{minimize}_{\boldsymbol{u} \in \overline{\mathscr{U}}, \chi \in \mathscr{\mathscr { \ell }}} \int_{\Omega} \bar{W}(\boldsymbol{E}, \boldsymbol{\chi}, \nabla \chi) d v+\Phi(\boldsymbol{u}),
$$

where $\overline{\mathscr{U}}$ is an appropriate open subset of $\mathscr{U}$. Let $\boldsymbol{p}_{q}=\nabla \chi_{q}$ and

$$
\begin{aligned}
\boldsymbol{S} & =\boldsymbol{F} \partial_{\boldsymbol{E}} \bar{W}(\boldsymbol{E}, \chi, \nabla \chi), \\
\pi_{q} & =-\partial_{\chi_{q}} \bar{W}(\boldsymbol{E}, \chi, \nabla \chi), \\
\boldsymbol{\xi}_{q} & =\partial_{\boldsymbol{p}_{q}} \bar{W}(\boldsymbol{E}, \chi, \nabla \chi),
\end{aligned}
$$

where $\partial_{\chi_{q}} \bar{W}$ is the " $q$-component" of the partial gradient with respect to $\chi$ of $\bar{W}$ on the hyperplane $\mathscr{P}$, while $\partial_{\boldsymbol{p}_{q}} \bar{W}$ is defined similarly (with (5.6) as the relevant constraint), so that the sums over $q$ of $\pi_{q}$ and of $\xi_{q}$ each vanish. We are then led to the more general Euler-Lagrange equations

$$
\operatorname{div} \boldsymbol{S}+\boldsymbol{b}=0, \quad \operatorname{div} \boldsymbol{\xi}_{q}+\pi_{q}=0 .
$$

Note that

$$
\sum_{q=1}^{Q}\left(\operatorname{div} \boldsymbol{\xi}_{q}+\pi_{q}\right)=0,
$$

so that only $Q-1$ of the balances $(5.16)_{2}$ are independent.

Acknowledgment. This work was supported by the National Science Foundation and by the Army Research Office.

Appendix. Formal derivation of the Euler-Lagrange equations. For convenience, we assume that the body force $b$ vanishes, so that the potential $\Phi(\boldsymbol{u})$ depends at most on the restriction of $\boldsymbol{u}$ to $\partial \Omega$.

a. The exact variational problem (3.2). Let $\boldsymbol{u}$ be a solution and restrict attention to a sufficiently small closed ball $B \subset$ (interior $\Omega$ ) such that the surface $\mathscr{S}$ across which $\boldsymbol{F}$ jumps divides $B$ into disjoint regions $B_{1}$ and $B_{2}$, with unit normal $\boldsymbol{n}$ to $\mathscr{S}$ outward from $B_{1}$. For all sufficiently small $t$, let $\hat{\boldsymbol{u}}(t) \in \mathscr{U}$ with $\hat{\boldsymbol{u}}(0)=\boldsymbol{u}$ and 
$\hat{\boldsymbol{u}}(t)-\boldsymbol{u}$ compactly supported in $B$, and assume that, in $B, \hat{\boldsymbol{u}}(t)$ jumps across a surface $\hat{\mathscr{S}}(t) \subset B$ that depends smoothly on $t$, has $\hat{\mathscr{S}}(0)=\mathscr{S} \cap B$, and has unit normal $\hat{\boldsymbol{n}}(t)$ with $\hat{\boldsymbol{n}}(0)=\boldsymbol{n}$. Let

$$
\widehat{W}(t)=\widetilde{W}(\widehat{\boldsymbol{E}}(t)), \quad \widehat{\boldsymbol{S}}(t)=\widetilde{\boldsymbol{S}}(\widehat{\boldsymbol{F}}(t)),
$$

with $\widehat{\boldsymbol{E}}(t)$ and $\widehat{\boldsymbol{F}}(t)$ the strain and deformation gradient corresponding to $\hat{\boldsymbol{u}}(t)$. Then

$$
\mathscr{V}(t):=\left\{\int_{B} \widehat{W}(t) d v\right\}^{\cdot} \text { satisfies } \mathscr{V}(0)=0,
$$

where the superscript dot denotes the derivative with respect to $t$. Let $\widehat{\mathscr{S}}^{\cdot}(t)$ denote the normal velocity of $\widehat{\mathscr{S}}(t)$ ( $t$ considered as time). Then

$$
\mathscr{V}=-\int_{\widehat{\mathscr{Y}}}[\widehat{W}] \widehat{\mathscr{S}}^{\cdot} d a+\int_{\widehat{B}_{1}} \widehat{\boldsymbol{S}} \cdot \widehat{\boldsymbol{F}}^{\cdot} d v+\int_{\widehat{B}_{2}} \widehat{\boldsymbol{S}} \cdot \widehat{\boldsymbol{F}}^{\cdot} d v
$$

$\left(\widehat{B}_{1}(t)\right.$ and $\widehat{B}_{2}(t)$ have obvious meanings), and integrating the second and third terms by parts yields

$$
\mathscr{V}=-\int_{\widehat{\mathscr{S}}}\left\{[\widehat{W}] \widehat{\mathscr{S}}^{\cdot}+\langle\widehat{\boldsymbol{S}}\rangle \hat{\boldsymbol{n}} \cdot[\hat{\boldsymbol{u}}]+[\widehat{\boldsymbol{S}}] \hat{\boldsymbol{n}} \cdot\langle\hat{\boldsymbol{u}} \dot{ }\rangle\right\} d a-\int_{\widehat{B_{1}}} \operatorname{div} \widehat{\boldsymbol{S}} \cdot \hat{\boldsymbol{u}} \cdot d v-\int_{\widehat{B}_{2}} \operatorname{div} \widehat{\boldsymbol{S}} \cdot \hat{\boldsymbol{u}} \cdot d v,
$$

where $\langle\boldsymbol{g}\rangle$ denotes the average of the limiting values of $\boldsymbol{g}$ on either side of $\widehat{\mathscr{S}}$. Thus, using the compatibility condition

$$
[\hat{\boldsymbol{u}}]=-\widehat{\mathscr{S}}^{\cdot}[\widehat{\boldsymbol{F}}] \hat{\boldsymbol{n}}
$$

and the fact that the "variations" $\hat{\boldsymbol{u}}$ ' and $\widehat{\mathscr{S}}$ ' are arbitrary, we find-upon setting $\mathscr{V}(0)=0-(3.4)$ with $b=0,(3.5)_{1}$, and

$$
[\widetilde{W}(\boldsymbol{E})-\widetilde{\boldsymbol{S}}(\boldsymbol{F}) \boldsymbol{n} \cdot \boldsymbol{F} \boldsymbol{n}]=0 ;
$$

but (3.3) and (3.5), yield $\widetilde{\boldsymbol{S}}(\boldsymbol{F}) \boldsymbol{n} \cdot \boldsymbol{F n}=\widetilde{\boldsymbol{S}}(\boldsymbol{F}) \cdot \boldsymbol{F}$, and $(3.5)_{2}$ follows.

$b$. The semi-quadratic variational problems (4.12), (4.15). We will establish only (4.15); (4.12) follows upon formally setting the $\sigma$ 's equal to zero.

Let $(\boldsymbol{u}, \boldsymbol{\chi})$ be a solution of (4.15). Let $B \subset$ (interior $\Omega$ ) be a sufficiently small closed ball such that $B$ intersects (only) $\Omega_{q}$ and $\Omega_{p}$, with $\mathscr{S}=\mathscr{S}_{q p} \subset B$ the corresponding interface. For all sufficiently small $t$, let $\hat{\boldsymbol{u}}(t) \in \mathscr{U}$ and $\hat{\chi}(t) \in \mathscr{Z}$ satisfy $\hat{\boldsymbol{u}}(0)=\boldsymbol{u}$ and $\hat{\boldsymbol{\chi}}(0)=\boldsymbol{\chi}$, and suppose that $\hat{\boldsymbol{u}}(t)-\boldsymbol{u}$ and $\widehat{\chi}(t)-\chi$ are compactly supported in $B$. Then, using notation analogous to that of the last section,

$$
\mathscr{V}(t):=\left\{\int_{B}(\widehat{W}(t)+\Gamma(\nabla \widehat{\chi}(t))) d v\right\}^{\cdot} \text { satisfies } \mathscr{V}(0)=0 .
$$

Further,

$$
\left\{\int_{B} \Gamma(\nabla \widehat{\chi}) d v\right\}^{\cdot}=\sigma_{q p}\{\operatorname{area}(\widehat{\mathscr{S}})\}^{\cdot}=-2 \sigma_{q p} \int_{\widehat{\mathscr{P}}} \widehat{K} \widehat{\mathscr{S}}^{\cdot} d a,
$$

so that steps analogous to (A3)-(A6) yield (4.16).

c. The regularized variational problems (5.9), (5.14). We will establish (5.14); (5.9) is a special case. Let $(\boldsymbol{u}, \boldsymbol{\chi})$ be a solution of (5.13). For all sufficiently small 
$t$, let $\hat{\boldsymbol{u}}(t) \in \overline{\mathscr{U}}$ and $\hat{\chi}(t) \in \overline{\mathscr{X}}$ satisfy $\hat{\boldsymbol{u}}(0)=\boldsymbol{u}$ and $\hat{\chi}(0)=\chi$, and suppose that $\hat{\boldsymbol{u}}(t)-\boldsymbol{u}$ and $\hat{\chi}(t)-\chi$ are compactly supported in $\Omega$. Then, using notation analogous to that of the preceding subsections,

$$
\mathscr{V}=\int_{\Omega}\left\{\widehat{\boldsymbol{S}} \cdot \widehat{\boldsymbol{F}}^{\cdot}+\sum_{q=1}^{Q}\left(\hat{\boldsymbol{\xi}}_{q} \cdot\left(\nabla \hat{\chi}_{q}\right)^{\cdot}-\hat{\pi}_{q}\left(\widehat{\chi}_{q}\right) \cdot\right)\right\} d v,
$$

and integrating $\widehat{\boldsymbol{S}} \cdot \widehat{\boldsymbol{F}}^{\cdot}$ and $\hat{\boldsymbol{\xi}}_{q} \cdot\left(\nabla \hat{\chi}_{q}\right)^{\cdot}$ by parts, we find that

$$
\mathscr{V}(0)=\int_{\Omega}\left\{\operatorname{div} \boldsymbol{S} \cdot \hat{\boldsymbol{u}}(0)-\sum_{q=1}^{Q}\left(\operatorname{div} \boldsymbol{\xi}_{q}+\pi_{q}\right)\left(\hat{\chi}_{q}\right)^{\cdot}(0)\right\} d v .
$$

But $\mathscr{V}(0)=0$. Thus, since $\hat{\boldsymbol{u}}^{(0)}$ is arbitrary, while $\left(\hat{\chi}_{q}\right)^{\circ}(0)$ must sum to zero, but is otherwise arbitrary, (5.16) follows.

\section{REFERENCES}

[1] A. G. Khachaturyan, Some questions concerning the theory of phase transformations in solids, Soviet Phys. Solid State 8, 2163-2168 (1967)

[2] A. G. Khachaturyan and G. A. Shatalov, Theory of macroscopic periodicity for a phase transition in the solid state, Soviet Phys. JETP 29, 557-561 (1969)

[3] A. L. Roitburd, The domain structure of crystals formed in the solid phase, Soviet Phys. Solid State 10, 2870-2876 (1969)

[4] A. L. Roitburd, Domain structure caused by internal stresses in heterophase solids, Phys. Status Solidi 16, 329-339 (1973)

[5] A. L. Roitburd and N. S. Kosenko, Orientational dependence of the elastic energy of a plane interlayer in a system of coherent phases, Phys. Status Solidi 35, 735-746 (1976)

[6] J. A. Wert, The strain energy of a disc-shaped GP zone, Acta Metall. Mater. 24, 65-71 (1976)

[7] A. L. Roitburd, Martensitic transformation as a typical phase transformation in solids, in Solid State Physics, vol. 33 Academic Press, 1979

[8] S. H. Wen, E. Kostlan, M. Hong, A. G. Khachaturyan, and J. W. Morris, The preferred habit of a tetragonal inclusion in a cubic matrix, Acta Metall. Mater. 29, 1247-1254 (1981)

[9] M. Hong, D. E. Wedge, and J. W. Morris, The state and habit of the $\mathrm{Fe}_{16} \mathrm{~N}_{2}$ precipiatae in b.c.c. iron: elastic theory, Acta Metall. Mater. 32, 279-288 (1984)

[10] E. Kostlan and J. W. Morris, The preferred habit of a coherent thin-plate inclusion in an anisotropic elastic solid, Acta Metall. Mater. 35, 745-777 (1987)

[11] R. V. Kohn, The relaxation of a double-well energy, Cont. Mech. Thermodyn. 3, 193-236 (1991)

[12] J. L. Ericksen, Some phase transitions in crystals, Arch. Rational Mech. Anal. 73, 99-124 (1980)

[13] R. Abeyaratne, Discontinuous deformation gradients away from the tip of a crack in anti-plane shear, J. Elast. 11, 373-393 (1981)

[14] J. L. Ericksen, Continuous martensitic transitions in thermoelastic solids, J. Thermal Stresses 4 , 107-119(1981)

[15] M. E. Gurtin and R. Temam, On the anti-plane shear problem in finite elasticity, J. Elast. 11, 197-206 (1981)

[16] R. D. James, Finite deformation by mechanical twinning, Arch. Rational Mech. Anal. 77, 143-176 (1981)

[17] R. Fosdick and G. MacSithigh, Helical shear of an elastic, circular tube with a non-convex stored energy, Arch. Rational Mech. Anal. 84, 31-53 (1983)

[18] M. E. Gurtin, Two-phase deformations of elastic solids, Arch. Rational Mech. Anal. 84, 1-29 (1983)

[19] R. Fosdick and G. MacSithigh, Minimization in incompressible nonlinear elasticity theory, J. Elast. 16, 267-301 (1986)

[20] R. D. James, Displacive phase transformations in solids, J. Mech. Phys. Solids 34, 359-394 (1986)

[21] J. M. Ball and R. D. James, Fine phase mixtures as minimizers of energy, Arch. Rational Mech. Anal. 100, 13-52 (1987) 
[22] I. Fonseca, Variational methods for elastic crystals, Arch. Rational Mech. Anal. 97, 189-220 (1987)

[23] R. D. James, The stability and metastability of quartz, in Metastablity and Incompletely Posed Problems (S. Antman, J. L. Ericksen, D. Kinderlehrer, and I. Müller, eds.), Springer-Verlag, 1987, pp. 147-175

[24] I. Fonseca, The lower quasiconvex envelope of the stored energy function for an elastic crystal, J. Math. Pures Appl. 67, 179-195 (1988)

[25] S. A. Silling, Consequences of the Maxwell relation for anti-plane shear deformations of an elastic solid, J. Elast. 19, 213-239 (1988)

[26] R. D. James and D. Kinderlehrer, Theory of diffusionless phase transformations, in Partial Differential Equations and Continuum Models of Phase Transitions (M. Rascle, D. Serre, and M. Slemrod, eds.), Lecture Notes in Phys., vol. 344, Springer-Verlag, Berlin, 1989, pp. 51-84

[27] K. Bhattacharya, Wedge-like microstructure in martensites, Acta Metall. Mater. 39, 2431-2444 (1990)

[28] P. Rosakis, Compact zones of shear transformation in an anisotropic solid, J. Mech. Phys. Solids 40, 1163-1195 (1992)

[29] E. Fried, Construction of two-phase equilibria in a non-elliptic hyperelastic material, J. Elast. 31, 71-123 (1993)

[30] P. Rosakis and H. Tsai, On the role of shear instability in the modelling of crystal twinning, Mech. Materials 17, 245-259 (1994)

[31] R. V. Kohn, The relationship between linear and nonlinear variational models of coherent phase transitions, in Transactions of the 7th Army Conference on Applied Mathematics and Computing (F. Dressel, ed.), 1989, pp. 279-304

[32] J. M. Ball and R. D. James, Proposed experimental tests of a theory of fine microstructure and the two-well problem, Philos. Trans. Roy. Soc. London A 338, 389-450 (1992)

[33] M. A. Grinfeld, Construction of a physically linear theory of coherent phase transformations, Mechanics of Solids 21, 84-96 (1986)

[34] M. E. Gurtin, An Introduction to Continuum Mechanics, Academic Press, New York, 1981

[35] E. Fried and M. E. Gurtin, Dynamic solid-solid transitions with phase characterized by an order parameter, Physica D 72, 287-308 (1994) 